\title{
Lutetium-labelled peptides for therapy of neuroendocrine tumours
}

\author{
B. L. R. Kam • J. J. M. Teunissen • E. P. Krenning • W. W. de Herder • S. Khan • \\ E. I. van Vliet • D. J. Kwekkeboom
}

Published online: 3 March 2012

(C) The Author(s) 2012. This article is published with open access at Springerlink.com

\begin{abstract}
Treatment with radiolabelled somatostatin analogues is a promising new tool in the management of patients with inoperable or metastasized neuroendocrine tumours. Symptomatic improvement may occur with ${ }^{177} \mathrm{Lu}$-labelled somatostatin analogues that have been used for peptide receptor radionuclide therapy (PRRT). The results obtained with ${ }^{177} \mathrm{Lu}$-[DOTA ${ }^{0}, \mathrm{Tyr}^{3}$ ] octreotate (DOTATATE) are very encouraging in terms of tumour regression. Dosimetry studies with ${ }^{177} \mathrm{Lu}$-DOTATATE as well as the limited side effects with additional cycles of ${ }^{177}$ Lu-DOTATATE suggest that more cycles of ${ }^{177}$ Lu-DOTATATE can be safely given. Also, if kidney-protective agents are used, the side effects of this therapy are few and mild and less than those from the use of ${ }^{90} \mathrm{Y}$-[DOTA ${ }^{0}, \mathrm{Tyr}^{3}$ ] octreotide (DOTATOC). Besides objective tumour responses, the median progression-free survival is more than 40 months. The patients' self-assessed quality of life increases significantly after treatment with ${ }^{177} \mathrm{Lu}$-DOTATATE. Lastly, compared to historical controls, there is a benefit in overall survival of several years from the time of diagnosis in patients treated with ${ }^{177} \mathrm{Lu}$-DOTATATE. These findings compare favourably with the limited number of alternative therapeutic approaches. If more widespread use of PRRT can be guaranteed, such therapy may well become the therapy of first choice in patients with metastasized or inoperable neuroendocrine tumours.
\end{abstract}

B. L. R. Kam $(\bowtie) \cdot$ J. J. M. Teunissen • E. P. Krenning • S. Khan •

E. I. van Vliet · D. J. Kwekkeboom

Department of Nuclear Medicine, Erasmus MC,

's Gravendijkwal 230,

3015 CE, Rotterdam, The Netherlands

e-mail: b.kam@erasmusmc.nl

W. W. de Herder

Department of Internal Medicine, Erasmus MC,

Rotterdam, The Netherlands
Keywords Lutetium · Somatostatin receptor · Peptide receptor radionuclide therapy (PRRT) $\cdot$ Lu-Octreotate . Neuroendocrine tumours

\section{Introduction}

In advanced and metastasized neuroendocrine tumours (NET), the use of surgery, external beam radiotherapy and chemotherapy as cytoreductive options is limited. The use of somatostatin analogues such as lanreotide and octreotide not only reduces hormonal overproduction resulting in symptomatic relief, but has also been shown to increase time to tumour progression in a placebo-controlled prospective study in patients with functional midgut neuroendocrine tumours treated with long-acting octreotide [1]. Peptide receptor scintigraphy in humans started with the demonstration of somatostatin receptor-positive tumours in patients using a radioiodinated somatostatin analogue [2]. Later, other radiolabelled somatostatin analogues were developed, and two of these subsequently became commercially available: $\left[{ }^{111} \mathrm{In}-\mathrm{DTPA}{ }^{0}\right]$ octreotide (Octreoscan) [3] and ${ }^{99 \mathrm{~m}} \mathrm{Tc}$-depreotide (Neotect). Newer positron emission tomography radiopharmaceuticals have been developed.

In the early 1990s, treatment with radiolabelled somatostatin analogues started in patients with NETs. Peptide receptor radionuclide therapy (PRRT) started initially with $\left[{ }^{111}\right.$ In-DTPA $\left.{ }^{0}\right]$ octreotide with promising results such as symptomatic disease control, but partial remissions were rare $[4,5]$. Lessons learned from these studies were that severe toxicities such as bone marrow suppression, and even myelodysplastic syndrome in patients treated with high dosages of $>100 \mathrm{GBq}$ ( $>3$ Gy bone marrow radiation dose), as well as renal insufficiency and transient liver toxicity may occur. 
The next generation of PRRT used a modified somatostatin analogue, $\left[\mathrm{Tyr}^{3}\right]$ octreotide, with a higher affinity for the somatostatin receptor subtype-2. Thereby, a different chelator, DOTA instead of DTPA, was used in order to ensure a more stable binding of the intended $\beta$-emitting radionuclide ${ }^{90} \mathrm{Y}$. Using this compound $\left({ }^{90} \mathrm{Y}\right.$-[DOTA ${ }^{0}, \mathrm{Tyr}^{3}$ ] octreotide (DOTATOC; OctreoTher, Novartis, Basel, Switzerland; Onalta, Molecular Insight Pharmaceuticals, Cambridge, MA), different phase 1 and phase 2 PRRT trials have been performed. Lastly ${ }^{177} \mathrm{Lu}$-based PRRT has been introduced into clinical practice. This review discusses these compounds and summarizes the results from their use.

\section{Radionuclide characteristics}

PRRT using ${ }^{111}$ In as the therapeutic radionuclide has rarely been successful in terms of objective tumour response, due to the small particle range of its auger electrons and therefore low tissue penetration. ${ }^{90} \mathrm{Y}$ is a more suitable radionuclide with the emission of $\beta$-particles with a maximum energy of 2.27 MeV and tissue penetration of $12 \mathrm{~mm}$. The half-life of $64 \mathrm{~h}$ is comparable with that of ${ }^{111}$ In. Dosimetric calculations are however difficult due to its pure $\beta$-emission and doses has to be estimated by the use of either ${ }^{86} \mathrm{Y}$ or ${ }^{111} \mathrm{In}$ as a surrogate or the application of alternative methods such as ${ }^{90} \mathrm{Y}$ PET or bremsstrahlung imaging [6].

Following the first paper in 1968 [7] on the diagnostic use of ${ }^{177} \mathrm{Lu}$ for bone imaging there was little interest in its $\beta$-emission characteristics for therapy until 1985 when Bard et al. [8] described the use of ${ }^{177} \mathrm{Lu}$ in the treatment of arthritis in rabbits. ${ }^{177} \mathrm{Lu}$ is a medium-energy $\beta$-emitter with a maximum energy of $0.5 \mathrm{MeV}$ and a maximal tissue penetration of $2 \mathrm{~mm}$. Its half-life is 6.7 days. ${ }^{177} \mathrm{Lu}$ also emits low-energy $\gamma$-rays at 208 and $113 \mathrm{keV}$ with $10 \%$ and $6 \%$ abundance, respectively, which allows scintigraphy and subsequent dosimetry with the same therapeutic compound. The shorter $\beta$-range of ${ }^{177} \mathrm{Lu}$ provides better irradiation of small tumours, in contrast to the longer $\beta$-range of ${ }^{90} \mathrm{Y}$ which allows more uniform irradiation in large tumours that may show heterogeneous uptake. This was illustrated in an animal model, in which a combination of ${ }^{90} \mathrm{Y}$ - and ${ }^{177} \mathrm{Lu}$-labelled somatostatin analogues demonstrated a better tumour response than the use of each radiolabelled analogue separately [9].

\section{Chelator and peptide}

For the coupling of the radionuclide and the somatostatin analogue in PRRT, the chelator DOTA $(1,4,7,10$ tetraazacyclododecane-1,4,7,10-tetra-acetic acid) is often used. Several studies on ${ }^{177}$ Lu-based PRRT have investigated the use of different somatostatin analogues, such as [DOTA ${ }^{0}$, $\mathrm{Tyr}^{3}$ ] octreotide (DOTATOC), $\left[\mathrm{DOTA}^{0}, \mathrm{Tyr}^{3}\right]$ octreotate (DOTATATE) and [DOTA $\left.{ }^{0}-1-\mathrm{Nal}^{3}\right]$ octreotide (DOTANOC) [10-13]. The somatostatin analogue [DTPA0, Tyr $^{3}$ ]octreotate differs from [DTPA0, $\mathrm{Tyr}^{3}$ ] octreotide only in that the Cterminal threoninol is replaced with threonine. However, subtle changes in the structure of the chelator and the use of a different radionuclide or peptide affects the binding affinities for the different somatostatin receptor subtypes (Table 1) [14]. In a comparison in patients, it was found that the uptake of radioactivity, expressed as a percentage of the injected dose of ${ }^{177}$ Lu-DOTATATE, was comparable with the use of ${ }^{177} \mathrm{Lu}$-DOTATOC in the kidneys, spleen and liver, but was three to four times higher in four out of five tumours [15]. Therefore, ${ }^{177}$ Lu-DOTATATE has a potential advantage because of the higher absorbed doses that can be achieved in most tumours without increases in the doses to potentially dose-limiting organs. Also, in tumours in the same patients in a therapeutic setting, we found that the residence times are in favour of ${ }^{177} \mathrm{Lu}$-DOTATATE in comparison with ${ }^{177} \mathrm{Lu}$-DOTATOC by a factor of 2.1 (Fig. 1) [16]. In contrast, Forrer et al. [17] demonstrated no difference in tumour uptake of ${ }^{111} \mathrm{In}$-DOTATATE and ${ }^{111} \mathrm{In}$-DOTATOC, whereas ${ }^{111} \mathrm{In}$ DOTATOC showed a higher tumour-to-kidney absorbed dose ratio. However, a low amount of peptide $(10 \mu \mathrm{g})$ was administered to the patients without concomitant amino acid infusion, whereas Esser et al. [16] used $200 \mu \mathrm{g}$ peptide with amino acid infusion, which corresponds exactly to the clinical therapeutic setting. Wehrmann et al. [11] compared the biodistribution of ${ }^{177}$ Lu-DOTATATE and ${ }^{177}$ Lu-DOTANOC in patients, and concluded that tumour uptake and absorbed doses were comparable for the two radioligands, whereas whole-body retention was lower for ${ }^{177}$ Lu-DOTATATE, and therefore the authors advocated the therapeutic use of ${ }^{177} \mathrm{Lu}$-DOTATATE, because a lower whole-body retention potentially implies a lower bone marrow toxicity.

\section{Treatment protocols}

Although most treatment protocols are much alike, minor differences do exist. All published studies on PRRT using ${ }^{177} \mathrm{Lu}$-based somatostatin analogues used diagnostic ${ }^{111}$ In-DTPA-octreotide (Octreoscan), ${ }^{68} \mathrm{Ga}$-DOTANOC or ${ }^{68} \mathrm{Ga}$-DOTATOC with sufficient tumour uptake as a patient inclusion criterion. Due to the need for kidney protection [18], most groups combined treatment with some form of amino acid infusion. The Rotterdam and Bad Berka groups use 2.5\% L-lysine and 2.5\% L-arginine in 1,000 ml, while the Basel group uses a 2,000-ml infusion of an amino acid solution comprising Ringer's lactated Hartmann solution, Proteinsteril (B. Braun Medical), HEPA 8\%, Mg 5-Sulfat (B. Braun Medical) as mentioned by Forrer et al. [20, 21] to inhibit 
Table 1 Affinity profiles for human somatostatin receptors 1-5 of a series of somatostatin analogues. Values are halfmaximal inhibitory concentrations (SEM) in nanomoles

Adapted from reference [14]

\begin{tabular}{|c|c|c|c|c|c|}
\hline \multirow[t]{2}{*}{ Peptide } & \multicolumn{5}{|c|}{ Somatostatin receptor } \\
\hline & 1 & 2 & 3 & 4 & 5 \\
\hline Somatostatin-28 & $5.2(0.3)$ & $2.7(0.3)$ & $7.7(0.9)$ & $5.6(0.4)$ & $4.0(0.3)$ \\
\hline Octreotide & $>10,000$ & $2.0(0.7)$ & $187(55)$ & $>1,000$ & $22(6)$ \\
\hline DTPA-octreotide & $>10,000$ & $12(2)$ & $376(84)$ & $>1,000$ & $299(50)$ \\
\hline In-DTPA-octreotide & $>10,000$ & $22(3.6)$ & $182(13)$ & $>1,000$ & $237(52)$ \\
\hline DOTA-[Tyr $\left.{ }^{3}\right]$ octreotide & $>10,000$ & $14(2.6)$ & $880(324)$ & $>1,000$ & $393(84)$ \\
\hline DOTA- $\left[\mathrm{Tyr}^{3}\right]$ octreotate & $>10,000$ & $1.5(0.4)$ & $>1,000$ & $453(176)$ & $547(160)$ \\
\hline DOTA-lanreotide & $>10,000$ & $26(3.4)$ & $771(229)$ & $>10,000$ & $73(12)$ \\
\hline Y-DOTA-[Tyr $\left.{ }^{3}\right]$ octreotide & $>10,000$ & $11(1.7)$ & $389(135)$ & $>10,000$ & $114(29)$ \\
\hline Y-DOTA-[Tyr $\left.{ }^{3}\right]$ octreotate & $>10,000$ & $1.6(0.4)$ & $>1,000$ & $523(239)$ & $187(50)$ \\
\hline Y-DOTA-lanreotide & $>10,000$ & $23(5)$ & $290(105)$ & $>10,000$ & $16(3.4)$ \\
\hline
\end{tabular}

tubular reabsorption of the radiopeptide. The HartmannHEPA solution typically contains $1 \%$ lysine, making the treatment protocol in terms of renal protection less effective as higher amounts of lysine lead to a greater reduction in renal uptake of radioactivity [21]. This is striking, as Imhof et al. [22] treated all patients from 1997 onwards with a coinfusion of $1,000 \mathrm{ml}$ physiological saline containing $20.7 \mathrm{mg} / \mathrm{ml} \mathrm{of}$ arginine and $20.0 \mathrm{mg} / \mathrm{ml}$ of lysine, which is more than in the Ringer's lactated Hartmann solution mentioned above. The Milan group [23] used $25 \mathrm{~g}$ of lysine in 1,000 $\mathrm{ml}$ saline infused over $4 \mathrm{~h}$, followed by an additional $12.5 \mathrm{~g}$ of lysine in $500 \mathrm{ml}$ saline over $3 \mathrm{~h}$ twice daily on days 2 and 3 after therapy. The recent report by the Gothenborg group [24] does not mention the use of any form of kidney protection at all.

Wehrmann et al. [11] from the Bad Berka group studied eight patients with metastasized NET who received therapy initially with ${ }^{177}$ Lu-DOTANOC (mean injected activity 5,515 $\mathrm{MBq}$, range 3,600-7,400 $\mathrm{MBq}$ ), but continued the therapy with ${ }^{177}$ Lu-DOTATATE in three patients (injected activity not mentioned) as well as 61 patients who had one to four cycles of solely ${ }^{177} \mathrm{Lu}$-DOTATATE (mean injected activity 5,534 MBq, range 2,500-7,400 MBq). The rationale for the choice of which therapeutic scheme in each patient was not clear. Forrer et al. used two cycles of

Fig. 1 Typical example of better tumour uptake of ${ }^{177}$ Lu-DOTATATE (left) than that of ${ }^{177}$ Lu-DOTATOC (right) in the tumour of a patient with a gastroenteropancreatic NET which results in a longer mean residence time (mean residence time ratio 2.4 in favour of DOTATATE in this example). Adapted from reference [16]
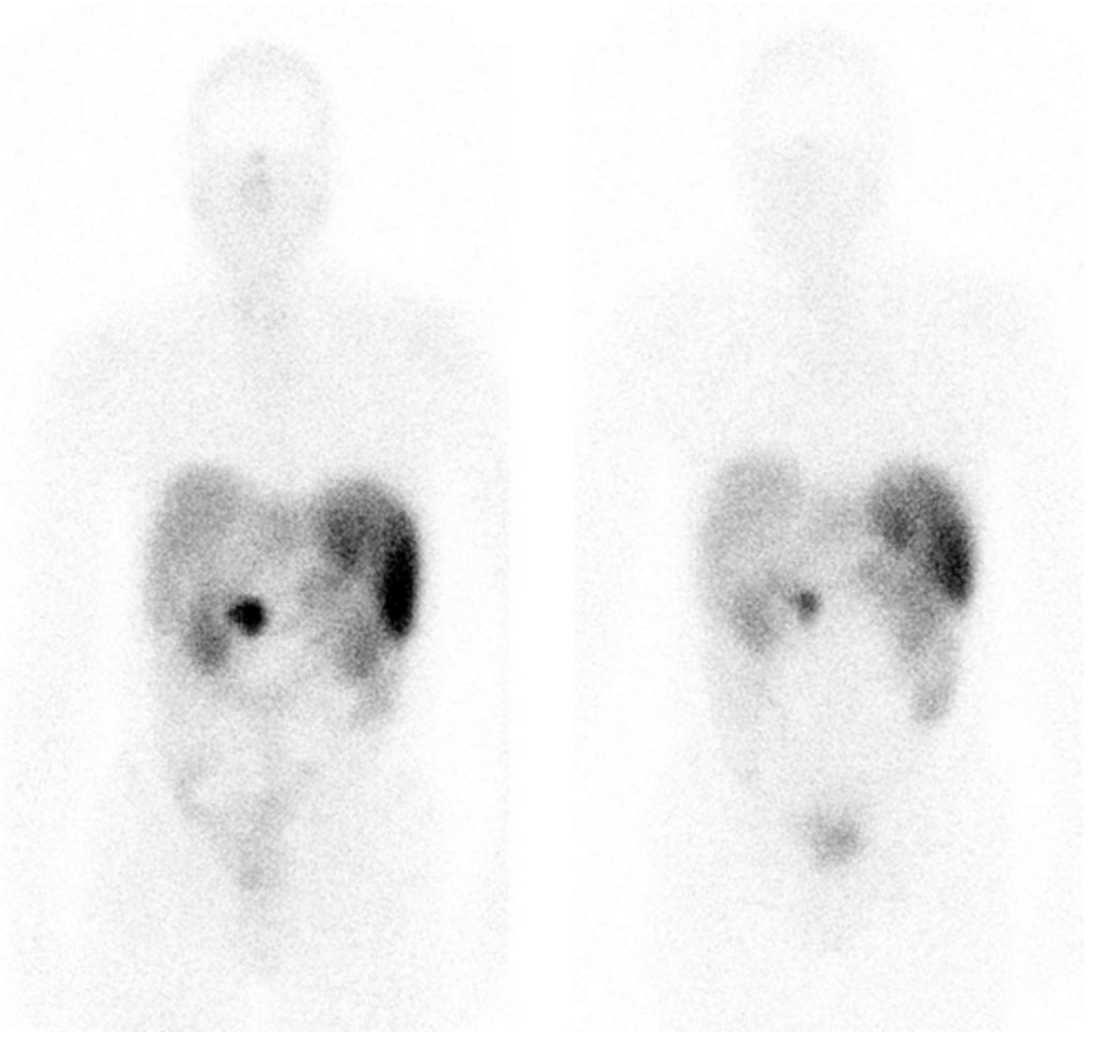
$7,400 \mathrm{MBq}{ }^{177} \mathrm{Lu}$-DOTATOC with an 8 -week interval in a study in 3 out of 28 patients with advanced paraganglioma and phaeochromocytoma and with predominantly small metastases $(<2 \mathrm{~cm})$ [20] after a single cycle of $3,700 \mathrm{MBq} / \mathrm{m}^{2}$ ${ }^{90}$ Y-DOTATOC. In the above-mentioned studies, it is impossible to ascertain the sole treatment effect of the ${ }^{177} \mathrm{Lu}$ labelled peptide. In another study by the same authors [21] and a further study by Frilling et al. [12] patients were treated with a single cycle of $7,400 \mathrm{MBq}{ }^{177} \mathrm{Lu}$-DOTATOC and those showing disease relapse after initial therapy with $7,400 \mathrm{MBq} / \mathrm{m}^{2}{ }^{90} \mathrm{Y}$-DOTATOC in two cycles. In a study by Kwekkeboom et al., patients who had not previously received PRRT were treated with 18.5 to $29.6 \mathrm{GBq}$ of ${ }^{177} \mathrm{Lu}$ DOTATATE usually in four cycles, to a maximum kidney dose of 23 Gy and a bone marrow dose of 2 Gy [15]. In a study by Bodei et al., patients were divided into two groups and received 3.7-5.18 GBq per cycle or 5.18-7.4 GBq per cycle with cumulative activities in the ranges 3.7-29.2 GBq and 5.55-28.9 GBq, respectively [23].

\section{Side effects}

Consistent with two previous reports $[25,26]$, the side effects of a maximum injected activity of 7,400 MBq per cycle of ${ }^{177}$ Lu-DOTATATE were analysed in 504 patients with gastroenteropancreatic (GEP) NETs [13]. Acute side effects occurring within $24 \mathrm{~h}$ after the administration of the radiopharmaceutical were nausea after $25 \%$ of administrations, vomiting after $10 \%$, and abdominal discomfort or pain after $10 \%$. Subacute haematological toxicity (WHO toxicity grade 3 or 4 ) occurred $4-8$ weeks after $3.6 \%$ of administrations or, expressed on a per-patient basis, after at least one of several treatments in $9.5 \%$ of patients. Factors associated with a higher frequency of haematological toxicity grade 3 or 4 were age over 70 years at treatment start, previous chemotherapy, creatinine clearance (estimated with Cockcroft's formula) $\leq 60 \mathrm{ml} / \mathrm{min}$, and the presence of bone metastases. When these factors were tested together in multivariate logistic regression, low creatinine clearance was a significant factor both for grade 3/4 thrombocytopenia $(p<0.001)$ and any haematological grade $3 / 4$ toxicity $(p<$ 0.001 ), whereas previous chemotherapy was less significant in predicting thrombocytopenia $(p<0.05)$. Creatinine clearance $\leq 60 \mathrm{ml} / \mathrm{min}$ was significantly more frequent in patients aged 70 years or more $(p<0.001$, Chi-squared test). Temporary hair loss (WHO grade 1; no baldness) occurred in $62 \%$ of patients. Serious delayed toxicities were observed in 9 of the 504 patients. There were two patients with renal insufficiency, which was probably unrelated to treatment with ${ }^{177}$ Lu-DOTATATE in both. There were three patients with serious liver toxicity, which was probably unrelated to treatment in both. Lastly, there were four patients with myelodysplastic syndrome, which was potentially treatmentrelated in three. In six patients with a highly hormonally active NET, a hormone-related crisis occurred after administration due to massive release of bioactive substances [27]. Bodei et al. [23] found no major acute or delayed renal or haematological toxicity (one grade 3 leukopenia and thrombocytopenia) in their cohort of 51 patients.

In three articles describing the use of ${ }^{177}$ Lu-DOTATOC [12, 20, 21], no serious side effects, including kidney toxicity, were reported. However, in these studies a maximum of $14.8 \mathrm{GBq}{ }^{177} \mathrm{Lu}$-DOTATOC was used in patients who had relapsed after earlier treatment with ${ }^{90} \mathrm{Y}$ - DOTATOC. The reported nausea and vomiting in $26 \%$ of all treated patients [15] were not reported separately in relation to ${ }^{90} \mathrm{Y}$ - or ${ }^{177} \mathrm{Lu}$-DOTATATE treatment. Currently, there are no reports of the side effects using ${ }^{177} \mathrm{Lu}$-DOTANOC.

\section{Dosimetry}

With the use of low-energy $\gamma$-rays at 208 and $113 \mathrm{keV}$ emitted by ${ }^{177} \mathrm{Lu}$, concomitantly with the therapeutic $\beta$-radiation, not only can posttreatment scans be acquired, but patient dosimetry can also be performed. The absorbed radiation doses to dose-limiting organs such as the kidneys and bone marrow are calculated to better tailor the total cumulative activity that can be administered to the individual patient. The absorbed radiation dose to the kidneys varies widely between patients treated with ${ }^{177}$ Lu-DOTATATE [15]. Based on calculations performed in six patients with a limited tumour load, the radiation dose to the bone marrow did not vary much between patients, and was estimated at a mean of $0.07 \mathrm{mGy} / \mathrm{MBq}$ (range $0.05-0.08 \mathrm{mGy} /$ $\mathrm{MBq}$ ). Therefore, allowing a maximum of $2 \mathrm{~Gy}$ for the absorbed dose to the bone marrow, the resulting cumulative dose that can be given was fixed at 29.6 GBq [15]. In a study by Werhmann et al. [11] in 69 patients the dose to the bone marrow was also variable between patients, and the calculated dose in most patients was $0.04 \pm 0.02 \mathrm{mGy} / \mathrm{MBq}$ (range 0.02 $0.08 \mathrm{mGy} / \mathrm{MBq}$ ). This was confirmed in another study in 15 patients by Forrer et al., in which the calculated dose was $0.03 \mathrm{mGy} / \mathrm{MBq}$ [28]. In the study by Bodei et al. [23], in 12 of 51 patients the calculated bone marrow dose was $0.03 \mathrm{mGy} /$ $\mathrm{MBq}$ with a cumulative dose in the range 0.5-1.3 Gy.

In addition, in $53 \%$ of 334 patients in whom unpublished kidney dosimetry was performed, an upper limit of 23 Gy for the absorbed dose to the kidneys would be reached with a total cumulative administered dose of $33.3 \mathrm{GBq}$ or more. This means that with fixed dose regimens that show relatively few side effects, a proportion of patients would be under-treated. A study by Garkavij et al. [29] demonstrated a large difference in calculated absorbed doses to the kidneys depending on the dosimetry method used. The dose varied from $1.15 \mathrm{mGy} / \mathrm{MBq}$ using the conventional planar 
abdominal background based method to $0.81 \mathrm{mGy} / \mathrm{MBq}$ using SPECT calculations, which suggests under-treatment if fixed maximal cumulative administered activities are used. Similar kidney dosimetry calculations were reported by Wehrmann et al. [11] and Bodei et al. [23]. Valkema et al. [30] reported a relatively mild decrease in creatinine clearance in patients treated with ${ }^{177} \mathrm{Lu}$-DOTATATE (median $3.8 \%$ per year) and a higher decrease in patients treated with

${ }^{90}$ Y-DOTATOC (median $7.3 \%$ per year), suggesting that higher administered cumulative activities of ${ }^{177}$ Lu-DOTATATE may be feasible. More important is the observation from this study that a creatinine clearance decrease of more than $25 \%$ per year is required for end-stage renal disease to develop within 5 years. Such a decrease in kidney function was found in only 1 of 37 patients treated with ${ }^{177} \mathrm{Lu}$-DOTATATE. Additionally, hypertension and diabetes were found to be risk factors for the development of kidney function loss after PRRT $[30,31]$. If these comorbidities are present, one would consider lowering the upper limit for the absorbed dose to the kidneys, as suggested by Bodei et al. [31]. Lastly, both the upper limits for the maximum absorbed dose to the kidneys accepted in PRRT and the calculation methods that are used for dosimetry may be questioned. The accepted upper limit for the dose to the kidneys of $23 \mathrm{~Gy}$ is derived from the experience of external beam irradiation, which uses much higher dose rates than PRRT. Also, the heterogeneous distribution of radioactivity in the kidneys after PRRT invalidates the MIRDOSE-based dosimetry models for low-energy emitting radionuclides such as ${ }^{177} \mathrm{Lu}[32]$. This is in contrast to the findings of Swärd et al. [24] of a significant decrease in glomerular filtration rate (GFR, mean $80 \pm 4 \mathrm{ml} / \mathrm{min}$ per $1.73 \mathrm{~m}^{2}$ to $70 \pm 4 \mathrm{ml} / \mathrm{min}$ per $1.73 \mathrm{~m}^{2}$ ) at an unknown point in time during follow-up after treatment with ${ }^{177}$ Lu-DOTATATE. This group, however, did not mention the use of any form of renal protection at all.

Because of the huge variation between patients and the limitations of the currently available methods for dosimetry after PRRT, individual dosimetry for the absorbed doses to both the bone marrow and the kidneys is desirable. Tailored or individual dosimetry, currently based on urine collection, repeated imaging and blood sampling after therapy, is timeconsuming and labour-intensive. Therefore, individualized calculations need to be simplified, and the need for a consensus as to the preferred method for optimal dosimetry is obvious. The maximum safe accumulated dose calculated using such an individualized method may be higher in a considerable number of patients and thereby this approach may also increase the maximum cumulative radiation dose to the tumour.

\section{Therapy response in PRRT-naive patients}

Kwekkeboom et al. analysed responses to ${ }^{177}$ Lu-DOTATATE treatment according to tumour type at 3 months after the last therapy cycle in 310 patients [13]. Patients were treated up to an intended cumulative activity of 22.2 29.6 GBq (600-800 mCi). The overall objective tumour response rate including complete remission (CR), PR and minor response (MR) was $46 \%$ (Table 2) (Fig. 2). Prognostic factors for predicting tumour remission (CR, PR or MR) as the treatment outcome were high uptake on diagnostic Octreoscan imaging $(p<0.01)$ and a Karnofsky performance score of $>70$ $(p<0.05)$. A small percentage of patients who had either stable disease (SD) or MR at their first two evaluations after therapy, i.e. 6 and 12 weeks after the last treatment cycle, had a further improvement in categorized tumour response at 6 months and 12 months, occurring in $4 \%$ of patients and $5 \%$ of patients, respectively. Three of four patients with clinically nonfunctioning neuroendocrine pancreatic tumours that were judged inoperable before treatment with ${ }^{177} \mathrm{Lu}$-DOTATATE, and who had a PR, were successfully operated on 6-12 months after their last treatment, but the fourth died of postoperative complications. In a small group of 21 patients treated with ${ }^{177} \mathrm{Lu}$ DOTATATE by Garkavij et al. [29], 12 were evaluated for objective response using RECIST criteria. PR was found in two patients, MR in three and SD in five. In the last study reported by Bodei et al. [23], 1 patient had CR, 14 had PR, 14 had MR, 14 had SD, and 9 had progressive disease (PD).

Another study evaluated the quality of life (QoL) in our first 50 Dutch patients with metastatic somatostatin receptorpositive GEP tumours treated with ${ }^{177}$ Lu-DOTATATE [33]. The patients completed the European Organization for the Research and Treatment of Cancer Quality of Life Questionnaire $\mathrm{C} 30$ [34] before therapy and during the follow-up visit 6 weeks after the last cycle. A significant improvement in the global health status/QoL scale was observed after therapy with ${ }^{177}$ Lu-DOTATATE. Furthermore, significant improvement was observed in emotional and social function scales. The symptom scores for fatigue, insomnia and pain decreased significantly. Patients with proven tumour regression most frequently had an improvement in QoL domains. However, because of the lack of a control group in this study, some placebo effect cannot be ruled out completely. This was also confirmed in a later study in 265 patients by Khan et al. [35], who demonstrated not only an improvement in QoL, but also in Karnofsky performance score. Furthermore, no decrease in QoL was found in patients without symptoms prior to PRRT.

There are no reports of the treatment outcome for ${ }^{177} \mathrm{Lu}$-DOTANOC or ${ }^{177} \mathrm{Lu}$-DOTATOC in patients who have not received PRRT.

\section{${ }^{177}$ Lu-DOTATATE versus other radiolabelled somatostatin analogues}

PRRT with ${ }^{177} \mathrm{Lu}$-DOTATATE is a promising new tool in the management of patients with inoperable or metastasized 
Table 2 Tumour responses in patients with NETs treated with different radiolabelled somatostatin analogues (adapted from reference [26])

\begin{tabular}{|c|c|c|c|c|c|c|c|}
\hline \multirow[t]{2}{*}{ Reference } & \multirow[t]{2}{*}{ Ligand } & \multirow{2}{*}{$\begin{array}{l}\text { Number } \\
\text { of patients }\end{array}$} & \multicolumn{5}{|c|}{ Tumour response } \\
\hline & & & $\mathrm{CR}$ & PR & MR & SD & $\mathrm{PD}$ \\
\hline [31] & ${ }^{90}$ Y-DOTATOC & 21 & 0 & $6(29 \%)$ & NA & $11(52 \%)$ & $4(19 \%)$ \\
\hline$[37,38]$ & ${ }^{90}$ Y-DOTATOC & 74 & $3(4 \%)$ & $15(20 \%)$ & NA & $48(65 \%)$ & $8(11 \%)$ \\
\hline [39] & ${ }^{90}$ Y-DOTATOC & 33 & $2(6 \%)$ & $9(27 \%)$ & NA & $19(57 \%)$ & $3(9 \%)$ \\
\hline$[40]$ & ${ }^{90}{ }^{Y-D O T A T O C ~}$ & 58 & 0 & $5(9 \%)$ & $7(12 \%)$ & $33(61 \%)$ & $10(19 \%)$ \\
\hline [13] & ${ }^{177} \mathrm{Lu}$-DOTATATE & 310 & $5(2 \%)$ & $86(28 \%)$ & $51(16 \%)$ & $107(35 \%)$ & $61(20 \%)$ \\
\hline [29] & ${ }^{177} \mathrm{Lu}$-DOTATATE & 12 & 0 & $2(17 \%)$ & $3(25 \%)$ & $5(42 \%)$ & $2(17 \%)$ \\
\hline$[23]$ & ${ }^{177}$ Lu-DOTATATE & 51 & $1(2 \%)$ & $14(27 \%)$ & $13(26 \%)$ & $14(27 \%)$ & $9(18 \%)$ \\
\hline
\end{tabular}

neuroendocrine tumours, and it is of interest to compare the results with those following the use of other radiolabelled somatostatin analogues. The analogue that has been used most often is ${ }^{90}$ Y-DOTATOC and the results with this analogue are also very encouraging, although a direct, randomized comparison between ${ }^{90} \mathrm{Y}$-DOTATOC and ${ }^{177} \mathrm{Lu}$-DOTATATE is lacking. The results of treatment with ${ }^{90} \mathrm{Y}$-DOTATOC in a large group of patients with various NETs (treated in Basel, Switzerland) have been reported [22]. If patients had a clinical or biochemical response (numbers/percentages not stated) or morphological disease control (morphological response or SD) after the first treatment cycle, additional cycles were given. Of 1,109 patients, $378(34.1 \%)$ showed a morphological response. However, this was not base on RECIST or SWOG criteria. SD was seen in 58 patients (5.2\%). Median survival from diagnosis was 94.6 months. Data on median progression-free survival were not given. Morphological, biochemical and clinical responses, and high tumour uptake on somatostatin receptor scintigraphy, were associated with longer median survival. Since morphological, biochemical and/or clinical responses were the criteria for treatment with additional cycles of ${ }^{90}$ Y-DOTATOC, a dose-effect relationship in terms of longer survival cannot be ruled out. An extremely high percentage of patients $(9.2 \%)$ experienced severe permanent renal toxicity (grade 4, GFR 15-29 $\mathrm{ml} / \mathrm{min}$ per $1.73 \mathrm{~m}^{2}$; or grade 5 , GFR $<15 \mathrm{ml} / \mathrm{min}$ per $1.73 \mathrm{~m}^{2}$ or dialysis) despite the use of renal protection with $20.7 \mathrm{mg} / \mathrm{ml}$ of arginine ( $2 \%$ arginine) and $20.0 \mathrm{mg} / \mathrm{ml}$ of lysine (2\% lysine) in $110.9 \% \mathrm{NaCl}$. However, it is not clear whether all study patients had received this combination. In earlier studies from the same group [36-38], the reports state that patients received the same Hartmann-HEPA solution, as used in the Lu-DOTATOCtreated patients from the same group.

Other reported percentages of tumour remission after ${ }^{90}$ Y-DOTATOC treatment vary (Table 2) [38-41]. There may be several reasons why different results were found in the centres performing trials with the same compound. First, there may be differences in the administered doses and dosing schemes. Some studies used dose-escalating schemes, whereas others used fixed doses. Second, there may be differences in patient and/or tumour selection. Several patient and tumour characteristics are prognostic for treatment outcome, such as amount of uptake on diagnostic Octreoscan imaging, estimated total tumour burden and the extent of liver involvement. Therefore, differences in patient selection may play an important role in determining treatment outcome.
Fig. 2 Typical example of a partial response (PR) in a patient with a NET of the small bowel with liver metastases treated with $29.6 \mathrm{GBq}$

${ }^{177}$ Lu-DOTATATE. a CT scan showing multiple liver metastases before treatment. b CT scan 6 weeks after treatment with regression of liver metastases, consistent with a PR
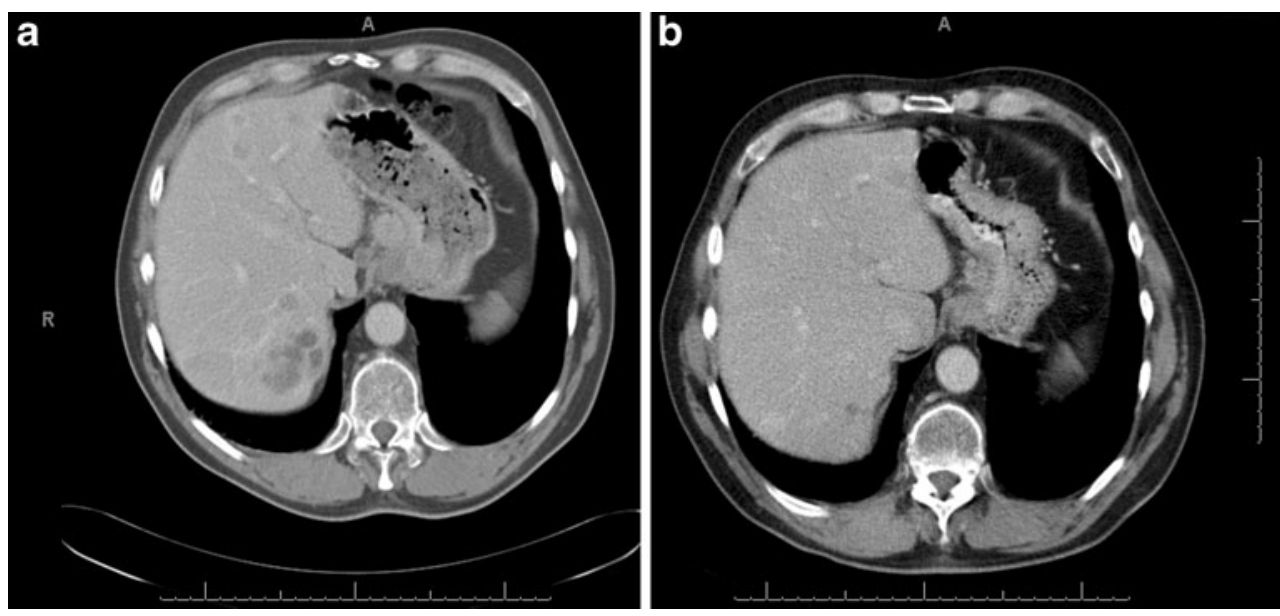
Third, there may be differences in tumour response criteria as some centres use the WHO tumour response criteria and others modified Southwest Oncology Group standard response criteria, with or without sonography for tumour size assessment. Finally, there may be centralized or decentralized follow-up CT scoring.

Our analysis in patients treated with ${ }^{177} \mathrm{Lu}$-DOTATATE indicated that the two most important prognostic factors for a favourable treatment outcome were high patient performance score and high uptake on pretreatment Octreoscan imaging. It is obvious that different studies can only be reliably compared if stratification for these factors is applied. From the published data, such a stratified comparison cannot be performed. Also, in order to establish which treatment scheme and which radiolabelled somatostatin analogues or combination of analogues is optimal, randomized trials are needed.

\section{Retreatment}

Because PRRT rarely results in CR, tumour progression will occur during follow-up. If the patient meets certain criteria mainly concerning bone marrow reserve, kidney function and tumour uptake on the diagnostic somatostatin receptor scan, retreatment may be an option. Forrer et al. [21] retreated 27 patients who had initially been treated with ${ }^{90}$ Y-DOTATOC (8 patients had $11,100 \mathrm{MBq} / \mathrm{m}^{2}, 19 \mathrm{had}$ $7,400 \mathrm{MBq} / \mathrm{m}^{2}$ in three and two cycles, respectively) with a single cycle of $7.4 \mathrm{GBq}^{177}$ Lu-DOTATOC. ${ }^{177}$ Lu-DOTATOC was chosen to avoid possible renal toxicity due to the further use of ${ }^{90} \mathrm{Y}$. Of these 27 patients, 14 had had a radiological response (at least PR according to WHO criteria) after the regular treatment with ${ }^{90}$ Y-DOTATOC. Benefit after ${ }^{177}$ Lu-DOTATOC was seen in 19 patients (70\%): 12 had SD, 5 had MR, and 2 had PR. None of the patients with PR after ${ }^{90}$ Y-DOTATOC treatment had PD after a single cycle of ${ }^{177} \mathrm{Lu}$-DOTATOC. The authors concluded that retreatment with ${ }^{177}$ Lu-DOTATOC is feasible, safe and efficacious without serious adverse events.

Recently, data on retreatment with two cycles of $7.4 \mathrm{GBq}$ of ${ }^{177} \mathrm{Lu}$-DOTATATE in 33 patients previously treated with 22.2- 29.6 GBq ${ }^{177} \mathrm{Lu}$-DOTATATE were reported [42]. Of these 33 patients, 28 had had a radiological response (at least MR) after the regular treatment with usually four cycles of ${ }^{177}$ Lu-DOTATATE, and 5 had experienced a significant clinical improvement. All had CT-assessed tumour progression before the start of retreatment. In 7 patients $(24 \%)$ renewed tumour size reduction was observed, and $7(24 \%)$ had SD at follow-up. No major side effects were observed during a median follow-up of 16 months. It was concluded that in the absence of treatment alternatives, this salvage therapy is safe and can be effective in selected patients.

\section{Options to improve PRRT}

From animal experiments it can be inferred that ${ }^{90}$ Y-labelled somatostatin analogues may be more effective in larger tumours, whereas ${ }^{177} \mathrm{Lu}$-labelled somatostatin analogues may be more effective in smaller tumours, but their combination may be even more effective [43]. Therefore, apart from comparisons between radiolabelled octreotate and octreotide, and between somatostatin analogues labelled with ${ }^{90} \mathrm{Y}$ and those labelled with ${ }^{177} \mathrm{Lu}$, PRRT with combinations of ${ }^{90} \mathrm{Y}$ and ${ }^{177}$ Lu-labelled analogues should also be evaluated.

Future directions to improve this therapy may also include the use of radiosensitizing chemotherapeutic agents. Chemosensitization with 5-fluorouracil (5-FU) in combination with ${ }^{90}$ Y-labelled antibody radioimmunotherapy is feasible and safe [44]. Also, chemosensitization with 5-FU combined with [ ${ }^{111}$ In-DTPA] octreotide treatment resulted in symptomatic response in $71 \%$ of patients with NETs [45], whereas other studies using only $\left[{ }^{111}\right.$ In-DTPA] octreotide treatment have shown such responses in lower percentages [4, 5]. Numerous trials on the effects of combined chemotherapy and (fractionated) external beam radiotherapy have been performed. In many of these, 5-FU was used. More recent trials used the prodrug of 5-FU, capecitabine, which has the advantage of oral administration. Also with the combination of radiotherapy and capecitabine, increased efficacy in terms of tumour growth control was reported if compared to radiotherapy as a single treatment modality [46]. If capecitabine is used at relatively low doses $\left(1,600-2,000 \mathrm{mg} / \mathrm{m}^{2}\right.$ per day), grade 3 haematological or other toxicity is rare $[46,47]$. For these reasons, after a pilot study to establish the safety of the combined therapy protocol [48], we started a randomized trial comparing treatment with ${ }^{177}$ Lu-octreotate with and without capecitabine in patients with GEP NETs. Also, attempts to improve the results of this type of therapy may focus on further reducing the radiation absorbed dose to normal tissues and organs, such as the kidneys and bone marrow, or at increasing the receptor density on the tumours, for instance via receptor upregulation. Both strategies may increase the therapeutic window.

Intraarterial treatment in selected patients with a predominant tumour load in the liver has been reported to be safe and effective. McStay et al. [49] used [ $\left.{ }^{90} \mathrm{Y}_{\text {-DOTA }}{ }^{0}, \mathrm{Tyr}^{3}\right]$ lanreotide administered via the hepatic artery (mostly $2 \times 1 \mathrm{GBq}$ ) to treat 23 patients with NETs, and 3 of these patients showed PR and 12 showed SD. However, 2 of the 3 patients with PR also had had concomitant embolization. Clinical improvement and a decrease in serum tumour markers were observed in $60 \%$ of the patients. Limouris et al. [50] used $\left[{ }^{111} \mathrm{In}-\mathrm{DTPA}^{0}\right]$ octreotide $(6.3 \mathrm{GBq}$ per injection and with a maximum of 12 injections per patient) to treat 17 patients, and 9 of these patients showed CR or PR. 
Lastly, Kratochwil et al. [51] found a fourfold higher uptake after intraarterial administration of $\left[{ }^{68} \mathrm{Ga}^{-D O T A}{ }^{0}, \mathrm{Tyr}^{3}\right]$ octreotide compared with intravenous administration in the same patients. Therefore, in selected patients this type of administration seems advantageous.

The use of PRRT as a neoadjuvant treatment has also been advocated in animal studies by Breeman et al. [52] who reported an increased survival in rats treated for 8 days with ${ }^{177} \mathrm{Lu}$-DOTATATE after infusion of $0.25 \times 10^{6}$ viable CA20948 cells into the portal vein, which mimics liver micrometastases. In humans, the use of PRRT in previously judged inoperable NETs has been described by Kaemmerer et al. [53] in a case report with PRRT used in a neoadjuvant setting before surgery, by Kwekkeboom et al. [13] who reported that PRRT enabled surgery in four patients in a large cohort of more than 500 patients, by Sowa-Staszczak et al. [54] in two of six patients treated in a neoadjuvant setting, and by Barber et al. [55] who found the same in five.

\section{Conclusion}

Treatment with radiolabelled somatostatin analogues is a promising new tool in the management of patients with inoperable or metastasized neuroendocrine tumours. Treatment with ${ }^{177} \mathrm{Lu}-$ labelled somatostatin analogues that have been used in PRRT may lead to symptomatic improvement. The results obtained with ${ }^{177} \mathrm{Lu}$-DOTATATE are very encouraging in terms of tumour regression. Dosimetry studies with ${ }^{177} \mathrm{Lu}$-DOTATATE as well as the limited side effects after ${ }^{177}$ Lu-DOTATATE retreatment suggest that there is room for more cycles of ${ }^{177} \mathrm{Lu}$-DOTATATE than currently used. Also, if kidney protective agents are used, the side effects of PRRT are few and mild, and less than from ${ }^{90}$ Y-DOTATOC. CR, PR or MR may be achieved in almost $50 \%$ of patients and the duration of the therapy response is more than 40 months. The patients' self-assessed QoL increases significantly after treatment with ${ }^{177} \mathrm{Lu}$-DOTATATE. Lastly, compared to historical controls, patients treated with ${ }^{177}$ Lu-DOTATATE show an increase in overall survival of several years from the time of diagnosis. These data compare favourably with the limited number of alternative treatment approaches. If more widespread use of PRRT can be guaranteed, such therapy may well become the therapy of first choice in patients with metastasized or inoperable NETs.

Conflicts of interest E.P. Krenning and D.J. Kwekkeboom are stockholders in Advanced Accelerator Applications (AAA). The remaining authors (B.L.R. Kam, J.J.M. Teunissen, W.W. de Herder, S. Khan, E.I. van Vliet) have no conflicts of interest.

Open Access This article is distributed under the terms of the Creative Commons Attribution Noncommercial License which permits any noncommercial use, distribution, and reproduction in any medium, provided the original author(s) and source are credited.

\section{References}

1. Rinke A, Muller HH, Schade-Brittinger C, Klose KJ, Barth P, Wied $\mathrm{M}$, et al. Placebo-controlled, double-blind, prospective, randomized study on the effect of octreotide LAR in the control of tumor growth in patients with metastatic neuroendocrine midgut tumors: a report from the PROMID Study Group. J Clin Oncol. 2009;27(28):4656-63.

2. Krenning EP, Bakker WH, Breeman WA, Koper JW, Kooij PP, Ausema L, et al. Localisation of endocrine-related tumours with radioiodinated analogue of somatostatin. Lancet. 1989;1(8632):242-4.

3. Balon HR, Goldsmith SJ, Siegel BA, Silberstein EB, Krenning EP, Lang $\mathrm{O}$, et al. Procedure guideline for somatostatin receptor scintigraphy with (111)In-pentetreotide. J Nucl Med. 2001;42(7):1134-8.

4. Valkema R, De Jong M, Bakker WH, Breeman WA, Kooij PP, Lugtenburg PJ, et al. Phase I study of peptide receptor radionuclide therapy with [In-DTPA] octreotide: the Rotterdam experience. Semin Nucl Med. 2002;32(2):110-22.

5. Anthony LB, Woltering EA, Espenan GD, Cronin MD, Maloney TJ, McCarthy KE. Indium-111-pentetreotide prolongs survival in gastroenteropancreatic malignancies. Semin Nucl Med. 2002;32(2):123-32.

6. Walrand S, Flux GD, Konijnenberg MW, Valkema R, Krenning EP, Lhommel R, et al. Dosimetry of yttrium-labelled radiopharmaceuticals for internal therapy: 86 Y or 90 Y imaging? Eur J Nucl Med Mol Imaging. 2011;38 Suppl 1:S57-68. doi:10.1007/s00259-011-1771-7.

7. O'Mara RE, McAfee JG, Subramanian G. Rare earth nuclides as potential agents for skeletal imaging. J Nucl Med. 1969;10(1):49-51.

8. Bard DR, Knight CG, Page-Thomas DP. Effect of the intraarticular injection of lutetium-177 in chelator liposomes on the progress of an experimental arthritis in rabbits. Clin Exp Rheumatol. 1985;3(3):237-42.

9. de Jong M, Breeman WA, Valkema R, Bernard BF, Krenning EP. Combination radionuclide therapy using $177 \mathrm{Lu}-$ and 90 Y-labeled somatostatin analogs. J Nucl Med. 2005;46 Suppl 1:13S-7S

10. Sward C, Bernhardt P, Johanson V, Schmitt A, Ahlman H, Stridsberg $\mathrm{M}$, et al. Comparison of [177Lu-DOTA0,Tyr3]-octreotate and [177Lu-DOTA0,Tyr3]-octreotide for receptor-mediated radiation therapy of the xenografted human midgut carcinoid tumor GOT1. Cancer Biother Radiopharm. 2008;23(1):114-20. doi:10.1089/ cbr.2007.0421.

11. Wehrmann C, Senftleben S, Zachert C, Muller D, Baum RP. Results of individual patient dosimetry in peptide receptor radionuclide therapy with 177Lu DOTA-TATE and 177Lu DOTA-NOC. Cancer Biother Radiopharm. 2007;22(3):406-16. doi:10.1089/ cbr.2006.325.

12. Frilling A, Weber F, Saner F, Bockisch A, Hofmann M, MuellerBrand J, et al. Treatment with (90)Y- and (177)Lu-DOTATOC in patients with metastatic neuroendocrine tumors. Surgery. 2006;140 (6):968-76. discussion 76-7.

13. Kwekkeboom DJ, de Herder WW, Kam BL, van Eijck CH, van Essen M, Kooij PP, et al. Treatment with the radiolabeled somatostatin analog [177 Lu-DOTA 0,Tyr3]octreotate: toxicity, efficacy, and survival. J Clin Oncol. 2008;26(13):2124-30.

14. Reubi JC, Waser B, Schaer JC, Laissue JA. Somatostatin receptor sst1-sst5 expression in normal and neoplastic human tissues using receptor autoradiography with subtype-selective ligands. Eur J Nucl Med. 2001;28(7):836-46.

15. Kwekkeboom DJ, Bakker WH, Kooij PP, Konijnenberg MW, Srinivasan A, Erion JL, et al. [177Lu-DOTAOTyr3]octreotate: comparison with [111In-DTPAo]octreotide in patients. Eur J Nucl Med. 2001;28(9):1319-25. 
16. Esser JP, Krenning EP, Teunissen JJ, Kooij PP, van Gameren AL, Bakker WH, et al. Comparison of [(177)Lu-DOTA(0),Tyr(3)] octreotate and [(177)Lu-DOTA(0),Tyr(3)]octreotide: which peptide is preferable for PRRT? Eur J Nucl Med Mol Imaging. 2006;33(11):1346-51. doi:10.1007/s00259-006-0172-9.

17. Forrer F, Uusijarvi $\mathrm{H}$, Waldherr $\mathrm{C}$, Cremonesi $\mathrm{M}$, Bernhardt $\mathrm{P}$, Mueller-Brand J, et al. A comparison of (111)In-DOTATOC and (111)In-DOTATATE: biodistribution and dosimetry in the same patients with metastatic neuroendocrine tumours. Eur J Nucl Med Mol Imaging. 2004;31(9):1257-62. doi:10.1007/s00259-0041553-6.

18. Rolleman EJ, Melis M, Valkema R, Boerman OC, Krenning EP, de Jong M. Kidney protection during peptide receptor radionuclide therapy with somatostatin analogues. Eur J Nucl Med Mol Imaging. 2010;37(5):1018-31. doi:10.1007/s00259-009-1282-y.

19. Rolleman EJ, Valkema R, de Jong M, Kooij PP, Krenning EP. Safe and effective inhibition of renal uptake of radiolabelled octreotide by a combination of lysine and arginine. Eur J Nucl Med Mol Imaging. 2003;30(1):9-15. doi:10.1007/s00259-002-0982-3.

20. Forrer F, Riedweg I, Maecke HR, Mueller-Brand J. Radiolabeled DOTATOC in patients with advanced paraganglioma and pheochromocytoma. Q J Nucl Med Mol Imaging. 2008;52(4):334-40.

21. Forrer F, Uusijarvi H, Storch D, Maecke HR, Mueller-Brand J. Treatment with $177 \mathrm{Lu}-D O T A T O C$ of patients with relapse of neuroendocrine tumors after treatment with 90Y-DOTATOC. J Nucl Med. 2005;46(8):1310-6.

22. Imhof A, Brunner P, Marincek N, Briel M, Schindler C, Rasch H, et al. Response, survival, and long-term toxicity after therapy with the radiolabeled somatostatin analogue [90Y-DOTA]-TOC in metastasized neuroendocrine cancers. J Clin Oncol. 2011;29 (17):2416-23.

23. Bodei L, Cremonesi M, Grana CM, Fazio N, Iodice S, Baio SM et al. Peptide receptor radionuclide therapy with $177 \mathrm{Lu}$-DOTATATE: the IEO phase I-II study. Eur J Nucl Med Mol Imaging. 2011;38 (12):2125-35

24. Sward C, Bernhardt P, Ahlman H, Wangberg B, Forssell-Aronsson $\mathrm{E}$, Larsson M, et al. [177Lu-DOTA0-Tyr3]-octreotate treatment in patients with disseminated gastroenteropancreatic neuroendocrine tumors: the value of measuring absorbed dose to the kidney. World J Surg. 2010;34(6):1368-72. doi:10.1007/s00268-009-0387-6.

25. Kwekkeboom DJ, Bakker WH, Kam BL, Teunissen JJ, Kooij PP, de Herder WW, et al. Treatment of patients with gastro-enteropancreatic (GEP) tumours with the novel radiolabelled somatostatin analogue [177Lu-DOTA(0),Tyr3]octreotate. Eur J Nucl Med Mol Imaging. 2003;30(3):417-22. doi:10.1007/s00259-002-1050-8.

26. Kwekkeboom DJ, Teunissen JJ, Bakker WH, Kooij PP, de Herder WW, Feelders RA, et al. Radiolabeled somatostatin analog [177Lu-DOTA0,Tyr3]octreotate in patients with endocrine gastroenteropancreatic tumors. J Clin Oncol. 2005;23(12):2754-62.

27. de Keizer B, van Aken MO, Feelders RA, de Herder WW, Kam $\mathrm{BL}$, van Essen M, et al. Hormonal crises following receptor radionuclide therapy with the radiolabeled somatostatin analogue [177Lu-DOTA0,Tyr3] octreotate. Eur J Nucl Med Mol Imaging. 2008;35(4):749-55. doi:10.1007/s00259-007-0691-z.

28. Forrer F, Krenning EP, Kooij PP, Bernard BF, Konijnenberg M, Bakker $\mathrm{WH}$, et al. Bone marrow dosimetry in peptide receptor radionuclide therapy with $[177 \mathrm{Lu}-\operatorname{DOTA}(0), \operatorname{Tyr}(3)]$ octreotate. Eur J Nucl Med Mol Imaging. 2009;36(7):1138-46. doi:10.1007/ s00259-009-1072-6.

29. Garkavij M, Nickel M, Sjogreen-Gleisner K, Ljungberg M, Ohlsson T, Wingardh K, et al. 177Lu-[DOTA0,Tyr3] octreotate therapy in patients with disseminated neuroendocrine tumors: analysis of dosimetry with impact on future therapeutic strategy. Cancer. 2010;116 (4 Suppl):1084-92. doi:10.1002/cncr.24796.

30. Valkema R, Pauwels SA, Kvols LK, Kwekkeboom DJ, Jamar F, de Jong M, et al. Long-term follow-up of renal function after peptide receptor radiation therapy with (90)Y-DOTA(0),Tyr(3)-octreotide and (177)Lu-DOTA(0), Tyr(3)-octreotate. J Nucl Med. 2005;46 Suppl 1:83S-91S.

31. Bodei L, Cremonesi M, Zoboli S, Grana C, Bartolomei M, Rocca $\mathrm{P}$, et al. Receptor-mediated radionuclide therapy with $90 \mathrm{Y}$ DOTATOC in association with amino acid infusion: a phase I study. Eur J Nucl Med Mol Imaging. 2003;30(2):207-16. doi:10.1007/s00259-002-1023-y.

32. Konijnenberg M, Melis M, Valkema R, Krenning E, de Jong M. Radiation dose distribution in human kidneys by octreotides in peptide receptor radionuclide therapy. J Nucl Med. 2007;48 (1):134-42.

33. Teunissen JJ, Kwekkeboom DJ, Krenning EP. Quality of life in patients with gastroenteropancreatic tumors treated with $[177 \mathrm{Lu}-$ DOTA0,Tyr3]octreotate. J Clin Oncol. 2004;22(13):2724-9. doi:10.1200/JCO.2004.10.01622/13/2724.

34. Aaronson NK. Assessment of quality of life and benefits from adjuvant therapies in breast cancer. Recent Results Cancer Res. 1993;127:201-10.

35. Khan S, Krenning EP, van Essen M, Kam BL, Teunissen JJ, Kwekkeboom DJ. Quality of life in 265 patients with gastroenteropancreatic or bronchial neuroendocrine tumors treated with [177Lu-DOTA0,Tyr3]octreotate. J Nucl Med. 2011;52 (9):1361-8.

36. Otte A, Herrmann R, Heppeler A, Behe M, Jermann E, Powell P, et al. Yttrium-90 DOTATOC: first clinical results. Eur J Nucl Med. 1999;26(11):1439-47.

37. Waldherr C, Pless M, Maecke HR, Schumacher T, Crazzolara A, Nitzsche EU, et al. Tumor response and clinical benefit in neuroendocrine tumors after 7.4GBq (90)Y-DOTATOC. J Nucl Med. 2002;43(5):610-6.

38. Waldherr C, Pless M, Maecke HR, Haldemann A, Mueller-Brand J. The clinical value of [90Y-DOTA]-D-Phe1-Tyr3-octreotide (90Y-DOTATOC) in the treatment of neuroendocrine tumours: a clinical phase II study. Ann Oncol. 2001;12(7):941-5.

39. Waldherr C, Schumacher T, Maecke HR, Schirp U, Forrer F, Nitzsche EU et al. Does tumor response depend on the number of treatment sessions at constant injected dose using 90YttriumDOTATOC in neuroen- docrine tumors? Eur J Nucl Med 2002; 29 (Suppl 1):S100

40. Valkema R, Pauwels S, Kvols LK, Barone R, Jamar F, Bakker $\mathrm{WH}$, et al. Survival and response after peptide receptor radionuclide therapy with [90Y-DOTA0,Tyr3] octreotide in patients with advanced gastroenteropancreatic neuroendocrine tumors. Semin Nucl Med. 2006;36(2):147-56.

41. Bodei L, Cremonesi M, Ferrari M, Pacifici M, Grana CM, Bartolomei $\mathrm{M}$, et al. Long-term evaluation of renal toxicity after peptide receptor radionuclide therapy with 90Y-DOTATOC and 177Lu-DOTATATE: the role of associated risk factors. Eur J Nucl Med Mol Imaging. 2008;35(10):1847-56. doi:10.1007/s00259-0080778-1.

42. van Essen M, Krenning EP, Kam BL, de Herder WW, Feelders RA, Kwekkeboom DJ. Salvage therapy with (177)Lu-octreotate in patients with bronchial and gastroenteropancreatic neuroendocrine tumors. J Nucl Med. 2010;51(3):383-90.

43. De Jong M, Valkema R, Jamar F, Kvols LK, Kwekkeboom DJ, Breeman WA, et al. Somatostatin receptor-targeted radionuclide therapy of tumors: preclinical and clinical findings. Semin Nucl Med. 2002;32(2):133-40.

44. Wong JY, Shibata S, Williams LE, Kwok CS, Liu A, Chu DZ, et al. A phase I trial of 90 Y-anti-carcinoembryonic antigen chimeric T84.66 radioimmunotherapy with 5-fluorouracil in patients with metastatic colorectal cancer. Clin Cancer Res. 2003;9(16 Pt 1):5842-52.

45. Kong G, Lau E, Ramdave S, et al. High-dose In-111 octreotide therapy in combination with radiosensitizing 5-FU chemotherapy 
for treatment of SSR-expressing neuroendocrine tumors. J Nucl Med. 2005;46 Suppl 2:151P.

46. Rich TA, Shepard RC, Mosley ST. Four decades of continuing innovation with fluorouracil: current and future approaches to fluorouracil chemoradiation therapy. J Clin Oncol. 2004;22 (11):2214-32. doi:10.1200/JCO.2004.08.009.

47. Dunst J, Reese T, Sutter T, Zuhlke H, Hinke A, KollingSchlebusch K, et al. Phase I trial evaluating the concurrent combination of radiotherapy and capecitabine in rectal cancer. J Clin Oncol. 2002;20(19):3983-91.

48. van Essen M, Krenning EP, Kam BL, de Herder WW, van Aken MO, Kwekkeboom DJ. Report on short-term side effects of treatments with $177 \mathrm{Lu}$-octreotate in combination with capecitabine in seven patients with gastroenteropancreatic neuroendocrine tumours. Eur J Nucl Med Mol Imaging. 2008;35(4):743-8. doi:10.1007/s00259-007-0688-7.

49. McStay MK, Maudgil D, Williams M, Tibballs JM, Watkinson AF, Caplin ME, et al. Large-volume liver metastases from neuroendocrine tumors: hepatic intraarterial 90Y-DOTA-lanreotide as effective palliative therapy. Radiology. 2005;237(2):718-26.

50. Limouris GS, Chatziioannou A, Kontogeorgakos D, Mourikis D, Lyra M, Dimitriou P, et al. Selective hepatic arterial infusion of In111-DTPA-Phe1-octreotide in neuroendocrine liver metastases. Eur J Nucl Med Mol Imaging. 2008;35(10):1827-37. doi:10.1007/s00259-008-0779-0.
51. Kratochwil C, Giesel FL, Lopez-Benitez R, Schimpfky N, Kunze $\mathrm{K}$, Eisenhut $\mathrm{M}$, et al. Intraindividual comparison of selective arterial versus venous $68 \mathrm{Ga}$-DOTATOC PET/CT in patients with gastroenteropancreatic neuroendocrine tumors. Clin Cancer Res. 2010;16(10):2899-905. doi:1078-0432.CCR-10-0004.

52. Breeman WA, Mearadji A, Capello A, Bernard BF, van Eijck CH, Krenning EP, et al. Anti-tumor effect and increased survival after treatment with [177Lu-DOTA0,Tyr3] octreotate in a rat liver micrometastases model. Int J Cancer. 2003;104(3):376-9. doi:10.1002/ ijc. 10952.

53. Kaemmerer D, Prasad V, Daffner W, Horsch D, Kloppel G, Hommann $\mathrm{M}$, et al. Neoadjuvant peptide receptor radionuclide therapy for an inoperable neuroendocrine pancreatic tumor. World J Gastroenterol. 2009;15(46):5867-70.

54. Sowa-Staszczak A, Pach D, Chrzan R, Trofimiuk M, Stefanska A, Tomaszuk M, et al. Peptide receptor radionuclide therapy as a potential tool for neoadjuvant therapy in patients with inoperable neuroendocrine tumours (NETs). Eur J Nucl Med Mol Imaging. 2011;38(9):1669-74. doi:10.1007/s00259-011-18358.

55. Barber TW, Hofman MS, Thomson BN, Hicks RJ. The potential for neoadjuvant peptide receptor chemoradionuclide therapy to render inoperable pancreatic and duodenal neuroendocrine tumours resectable. Eur J Surg Oncol. 2012;38(1):64-71. doi:10.1016/j.ejso.2011.08.129 